\title{
An analysis of misleading YouTube videos on urological conditions: what to do about the danger of spreading misinformation of the YouTube videos?
}

\author{
Ismail Selvi ${ }^{1}$ (D) Numan Baydilli ${ }^{2}$ (i) \\ Received: 18 January 2021 / Accepted: 30 January 2021 / Published online: 9 February 2021 \\ (c) The Author(s), under exclusive licence to Springer-Verlag GmbH, DE part of Springer Nature 2021
}

Keywords Misleading information $\cdot$ Social media $\cdot$ Urology $\cdot$ YouTube

\section{Dear Editor,}

We read with great interest the valuable article by Pratsinis et al. [1] in a recent issue of the journal. YouTube is expected to disseminate updated medical information and guide patients to easily access useful knowledge. Contrary, as mentioned by the authors, it is seen that most viewed videos may not contain accurate information. People who present biased information with a commercial concern make some tricks to increase the discoverability and viewership of their videos [2]. This may lead to increase dissemination of misinformation. The previous studies of the authors on this subject have also emphasized that this problem is not only in the surgical treatment of urinary stones but also in other urological conditions [3].

In our recent study, we have assessed whether Youtube videos are reliable additional information sources for recommendations about what to do in the COVID-19 period when various urological symptoms are encountered [4]. Our analysis showed that median reliability and quality scores of all videos were high, and $77.2 \%$ of the videos were uploaded by healthcare providers (e.g. universities, professional organizations, non-profit physician/physician groups). Nevertheless, we observed similar findings to Pratsinis et al. [1] that the number of "views per day" and "likes" rates of videos with

This comment refers to the article available online at https://doi. org/10.1007/s00345-020-03236-6.

Ismail Selvi

ismselvi33@hotmail.com

1 Department of Urology, Başakşehir Çam and Sakura City Hospital, 34480 Istanbul, Turkey

2 Department of Urology, Erciyes University Medical School, Kayseri, Turkey misleading information were higher although they constituted only $14.7 \%$ of all videos. We observed that majority of the videos $(63.9 \%)$ contain andrological information, whereas videos containing uro-oncological information were $19.1 \%$. Although the highest rates of reliable information were found in videos about uro-oncology, we observed that these videos did not reach high uploads, views and likes rates as the videos related to andrology. This result indicates that the topic of sexual health makes people more curious even in the COVID-19 period. It also shows that andrological disorders are more susceptible to abuse due to the more possibility of misleading information dissemination. This study found that uro-oncology-related videos provide more reliable and better quality information among all videos, but less popular.

In our another study, we assessed the videos which have general information about testicular self-examination (TSE), and that contain the steps of TSE [2]. Among the first 300 most viewed videos, we found that 112 videos were relevant to our inclusion criteria. Although $63.4 \%$ of the included 112 videos contain useful informations, ideal videos with useful information actually make up 26\% of the 300 most viewed YouTube videos. This shows that it is not easy and practical for a layman to find a suitable video for the purpose by searching for "keyword" in the YouTube list.

As a contribution to valuable opinions of Pratsinis et al. [1], we consider that healthcare providers should give enough information at diagnosis to reduce the need for patients to search for additional information on social media or YouTube. Because it has been stated that $75 \%$ of patients do not consider the reliability of information source while searching the internet [2]. In addition, patients can be recommended YouTube channels of international or national health organizations (e.g. the EAU Patient Information 
webpage, as the authors point out [1]) through both healthcare providers and general public information spots.

Author contributions SI: Conception and design, Acquisition of data, Analysis and Interpretation of data, Drafting of the manuscript, Administrative, technical, or material support; BN: Conception and design, Critical revision of the manuscript for important intellectual content, Supervision.

Funding There is no financial support for this study.

\section{Compliance with ethical standards}

Conflict of interest The authors declare no conflicts of interest and has nothing to disclose.

Ethical approval This article does not contain any studies with human participants or animals performed by any of the authors.

\section{Reference}

1. Pratsinis M, Abt D, Müllhaupt G et al (2020) Systematic assessment of information about surgical urinary stone treatment on
YouTube. World J Urol. https://doi.org/10.1007/s00345-02003236-6 (Online ahead of print)

2. Selvi I, Baydilli N, Akinsal EC (2020) Can YouTube English videos be recommended as an accurate source for learning about testicular self-examination? Urology 145:181-189. https://doi. org/10.1016/j.urology.2020.06.082

3. Betschart P, Pratsinis M, Müllhaupt G, Rechner R, Herrmann TR, Gratzke C, Schmid HP, Zumstein V, Abt D (2020) Information on surgical treatment of benign prostatic hyperplasia on YouTube is highly biased and misleading. BJU Int 125(4):595-601. https:// doi.org/10.1111/bju.14971

4. Selvi I, Baydilli N (2021) Can YouTube video on urological problems be used as an additional resource in telemedicine during COVID-19 pandemic? J Urol Surg. https://doi.org/10.4274/jus. galenos.2020.0007 (Online ahead of print)

Publisher's Note Springer Nature remains neutral with regard to jurisdictional claims in published maps and institutional affiliations. 